\title{
Circuit
}

Musiques contemporaines

\section{Le métier : composer la musique contemporaine}

\section{Marcelle Guertin}

Volume 5, numéro 1, 1994

Gilles Tremblay : réflexions

URI : https://id.erudit.org/iderudit/902082ar

DOI : https://doi.org/10.7202/902082ar

Aller au sommaire du numéro

Éditeur(s)

Les Presses de l'Université de Montréal

ISSN

1183-1693 (imprimé)

1488-9692 (numérique)

Découvrir la revue

Citer cet article

Guertin, M. (1994). Le métier : composer la musique contemporaine. Circuit, 5(1), 10-18. https://doi.org/10.7202/902082ar d'utilisation que vous pouvez consulter en ligne.

https://apropos.erudit.org/fr/usagers/politique-dutilisation/ 


\section{Composer la musique contemporaine}

Propos recueillis par Marcelle Guertin, Dérives, nos 44-45, 1984, pp. $16-24^{(2)}$.

\section{Composer en 1984 à Montréal}

Composer, c'est mettre en relation des idées et, en même temps, être à l'écoute d'une poussée de pensées sonores qui vont du cri de l'enfant jusqu'aux pleurs ou au rire. II y a une très grande proximité entre ces pulsions instinctives et la composition, même si celle-ci est organisée par l'intelligence. La composition reste très près du vécu viscéral et mental. En ce sens, composer en 1984, c'est à peu près la même chose qu'en 1684, ou antérieurement, en l'an 5000 av. J.-C..

Toutefois, les fonctions ont changé historiquement et composer en 1984, c'est être conscient de l'état du monde en cette année 1984, comme composer en 1684, c'était peut-être être conscient, plus ou moins, de l'état du monde à ce moment-là. Je conçois très bien que des compositeurs puissent s'abstraire du temps et de l'espace, mais personnellement, je crois beaucoup à cette conscience d'un temps dans lequel on vit, d'un temps qui contient son drame. Je pense aussi qu'on peut en même temps s'en détacher. J'ai l'air de me contredire, mais je parle évidemment d'un détachement qui fait qu'on n'est pas esclave d'une situation. Je pense en particulier à Mozart, à qui je me réfère souvent comme à un très grand modèle éthique en composition. Voilà, on se dit: Mozart a vécu tel événement tragique et il a ensuite écrit un aria ou un concerto d'une joie absolument délirante. C'est vrai, mais ça ne veut pas forcément dire qu'il n'était pas conscient de la vie de son époque; il savait s'en détacher, la transcender. L'art est un bondissement de liberté. Ce bondissement est possible avec un certain détachement, mais d'autre part, le piédestal de ce détachement c'est la vie telle qu'elle est, telle qu'on la perçoit, et l'on ne peut s'en abstraire. Mais l'intérêt de l'art, c'est le dépassement. $C^{\prime}$ 'est, au fond, ce qui suscite l'enthousiasme. Les Grecs appellent ça la catharsis. On peut l'appeler l'extase (c'est un mot dont je n'ai pas peur), et les moments de plus grande joie quand je compose sont ceux où j'oublie totalement qui je suis. Mais totalement, comme un fou. Je crois que c'est une chose
(2) Lla revue Dérives a été publiée à Montréal de 1975 à 1988 sous la direction de Jean Jonassaint.) 
que tout le monde peut connaître dans différentes circonstances, bien que ce soit assez rare tout de même.

Il y a, pour moi, dans l'acte de créer - je n'aime pas tellement le mot création parce que je pense que l'homme poursuit la création ou révèle des créations - un aspect qui est très près du sacré. Dans toute œuvre, même la plus profane, cet aspect est présent, en ce sens que l'acte créateur appelle un émerveillement: autant chez celui qui fait l'œuvre que chez l'auditeur qui entrera en contact avec elle. Or, celui qui fait l'œuvre est en même temps fait par cet émerveillement, et je pense que c'est là l'essentiel des raisons qui m'ont attiré vers des thèmes religieux, comme dans Souffles ou Oralléluiants. J'ai trouvé dans certains psaumes, notamment ceux dits de David - qui sont d'une grande poésie et où il y a une sorte de parallèle avec l'acte même de créer -, cet éblouissement de l'émerveillement. Je ne peux dissocier l'acte créateur et le sacré : il y a partage de l'étonnement avec toutes les manifestations alléluiantes, qu'elles soient humaines ou naturelles: les chutes d'eau, les chants d'oiseaux, le chant grégorien, la musique africaine ou hindoue... il y a une sorte de mouvement alléluiant qui enveloppe tout cela. Qu'on soit religieux ou non n'a pas d'importance, l'émerveillement me paraît être à la source de tout, à la base de toute vie possible entre les êtres.

Maintenant: est-ce que ça a une incidence de composer à Montréal ? Sûrement. Composer à Tombouctou, à Bali, dans les pays nordiques, sous les tropiques nous met dans des environnements tellement différents que ça crée nécessairement des conditionnements qui sont différents et qui donneront lieu à des tempi plus ou moins rapides, plus ou moins contemplatifs, quoiqu'il n'y ait rien de systématique. Mais, il y a quand même un conditionnement quand on vit dans un pays chaud, dans un pays froid, et surtout, lorsqu'on vit dans un centre culturel bouillonnant ou dans un désert culturel.

Pour revenir en 1984, à Montréal, je dirais qu'il y a une sorte de courbe: je sens actuellement, avec un certain pessimisme, avec une certaine angoisse même, une courbe descendante. II y a eu une côte ascendante autour des années 1970-1975, mais maintenant, il me semble avoir en Amérique du Nord, et en Europe également, un recul politique. Ce que je veux dire, c'est qu'il y a une tendance fascisante grandissante dans le monde, et l'art s'en ressent beaucoup: l'artiste authentique me semble de plus en plus à contre-courant. 


\section{Le point de départ d'une composition}

Il y a des œuvres pour lesquelles je ne sais pas comment les idées sont arrivées. D'une façon générale, je peux dire que pour chaque pièce, le point de départ est différent, et le processus de composition également. II y a des œuvres qui naissent à partir d'un geste musical: une phrase, une sonorité, un enchaînement; d'autres qui sont engendrées de façon globale, par une idée. C'est un peu le cas de Solstices. II y a des œuvres qui s'élaborent de façon linéaire; d'autres qui sont soumises à une constellation d'idées: ces idées, faites d'autant d'étoiles, s'entrechoquent, se mettent à avoir des relations les unes avec les autres et l'œuvre essaie de trouver un point d'équilibre entre tout ça. Je parle de façon un peu abstraite. Pour Solstices, c'est beaucoup plus simple. (En général, je n'aime pas parler des choses qui me touchent trop profondément, mais pour ce qui est de l'origine de cette œuvre, je peux en parler avec plus d'aisance car c'est une pure anecdote). Quelques mois avant de commencer la pièce, j'ai participé à une émission radiophonique en multiplex entre Paris, Bruxelles, Montréal et Genève aussi, je crois. C'était une émission organisée par Radio-France. II y avait une table ronde à Paris, deux ou trois personnes à Bruxelles et j'étais seul à Montréal. On m'avait dit: rendez-vous à telle heure, dans tel studio à Radio-Canada, c'est une émission en direct. Notre animateur était Georges Charbonnier. On a pris contact vingt minutes avant l'émission et au début, j'étais un peu intimidé, enfin, je me sentais vraiment tout seul dans mon petit studio à Radio-Canada; je me disais: "Qu'est-ce que je fais ici ? Comment va-ton faire pour communiquer $Z$ ». Je vous assure qu'au bout de cinq minutes, j'avais complètement oublié que j'étais à Montréal dans un studio, au mois de février, qu'il faisait froid, qu'il y avait de la neige; j'étais vraiment dans un autre lieu et je pouvais poser des questions directement à ceux qui étaient là-bas comme eux pouvaient le faire à mon adresse. Voilà un bel exemple de ce qu'on peut faire avec la technologie. Jusque là, me dira-t-on, on n'est pas dans la musique. Bien, mais à la fin de cette émission, moi j'étais tout à fait dans la musique: ça avait été une émission de conversations dans laquelle il n'y avait pas eu de musique, mais ces conversations étaient très musicales et on pouvait communiquer d'un lieu à un autre, provoquer des réactions, des réflexes, etc.; à ce moment-là, j'ai eu l'idée d'une œuvre où chaque instrument mènerait le bal pendant un certain temps et créerait des réactions chez les autres instruments qui lui répondraient avec des codes de questions et de réponses musicales. Bien sûr, ça a été adapté, ça a été greffé ensuite à l'idée des saisons: l'œuvre changerait de caractère selon qu'elle est jouée près du solstice d'hiver où elle est beaucoup 
plus calme, ou près du solstice d'été où elle sera exubérante. C'est une pièce pour six instruments, qui peut être jouée à plusieurs groupes. Jusqu'à présent, on l'a fait avec un groupe, puis avec deux groupes qui se répondent dans la même salle. Mais l'état ultime, c'est qu'elle soit exécutée dans des lieux différents, à l'aide de communications Telstar. L'œuvre pourrait commencer à Londres par exemple; à un signal - un peu comme dans les courses de relais -, le relais serait donné à Rome; à un autre signal, ça reviendrait à Londres puis on pourrait passer la parole à Montréal, puis réaliser un duo entre Rome et Montréal pendant qu'il y a une grande activité à Paris. Je n'ai pas osé faire intervenir plus de quatre villes dans mon projet, mais c'est possible. D'ailleurs, toute l'explication des règles de jeu figure dans la partition. Solstices n'a jamais été exécuté sous cette forme de relais Telstar et il n'y a que la radio qui puisse faire ça. Bien sûr, ça créerait certains problèmes techniques dans le sens où, lorsque les communications s'établissent entre des lieux éloignés, il y a un délai; un délai d'une fraction de seconde, mais qui, à un moment donné, peut créer des problèmes au point de vue rythmique. Par contre, c'est une fraction de seconde qu'on peut corriger par la technique également. Je parlais justement de ces problèmes avec un compositeur québécois qui habite Vancouver, Jean Piché(3), qui avait déjà fait une expérience semblable avec les Japonais. Ils se sont servi du satellite Telstar en exploitant musicalement et la réverbération provoquée, et le délai de communication. Au point de vue musical, c'était très exploratoire, et le fait de se servir d'un satellite comme chambre d'écho, était amusant.

\section{La prise en considération des interprètes et des problèmes d'exécution dans la composition}

La connaissance d'un interprète, des dons particuliers d'un interprète, peut influencer le compositeur. Ainsi lorsque le pianiste Claude Helffer, cet interprète merveilleux, m'a commandé une œuvre, je ne me suis pas privé de virtuosité parce que je savais qu'il pouvait faire tout ce que j'écrirais.

Les interprètes sont souvent de grands inspirateurs. J'ai travaillé avec un flûtiste puis avec un clarinettiste qui m'ont fait découvrir des aspects inouïs de leur instrument. Pour composer Dzei, une œuvre qui est centrée sur la clarinette basse, i'ai demandé à Nicolas Desjardins de venir à la maison, et pendant quelques jours, on a travaillé ensemble. Je lui ai dit: « Je voudrais savoir ce qu'on peut faire avec les sons de zone». II m'a joué des sons de zone, ces amalgames de sons et de bruits résultant de l'émission de notes
(3) Jean Piché est aujourd'hui professeur à la Faculté de musique de l'Université de Montréal (N.d.E.). 
fondamentales, ef il m'a montré des choses que je ne connaissais pas, et j'ai noté. C'est une collaboration qui est précieuse. En ce sens, l'interprète a, pour moi, un très grand rôle dans la composition, d'autant plus qu'il est un porteparole. J'admire le côté « honnêteté » de certains interprètes. Malheureusement, je ne peux pas dire cela de tous, puisque j'ai eu quelquefois des exécutions complètement ratées, sabotées, par manque de professionnalisme; c'était pourtant des professionnels qui jouaient, mais ils n'avaient aucun souci professionnel. À côté de cela, il y a des interprètes qui mettent un tel soin dans l'exécution! C'est un plaisir de travailler avec eux; on a vraiment l'impression qu'on bâtit ensemble.

Dans certaines de mes œuvres, il y a des jeux - dans le sens ludique du terme -, donc un côté improvisation. Je crois que c'est une chose qui est intéressante pour l'interprète et qui peut lui donner la possibilité d'interpréter encore davantage - pour donner un exemple ancien, on peut comparer cela à la cadenza non écrite de certains concertos. Ça peut être assez amusant à faire également, particulièrement dans le cas de jeux de réflexes entre musiciens: comme tout se déroule très vite, les réflexes manquent souvent ou viennent en retard. (C'est comme lorsqu'on joue à se taper sur la main : à un moment donné, on n'enlève pas la main assez vite et on se fait taper dessus). Ce type de jeu est difficilement prévisible par le compositeur mais ce que j'aime dans ces situations, c'est que ça crée un suspense rythmique dans lequel l'erreur possible est aussi intéressante que la réussite.

\section{Enseigner la composition instrumentale}

On n'enseigne pas la composition. Tout ce qu'on peut faire, c'est parler technique. Quand, par exemple, quelqu'un écrit trop grave pour un piccolo ou trop haut pour une contrebasse, on dit: "Ça ne marche pas parce que l'instrument est incapable de le faire ». Quand une chose n'est pas claire, on demande: "Pourquoi avez-vous écrit ça de cette façon plutôt que d'une autre, car si moi je ne comprends pas, comment voulez-vous que l'interprète qui va lire votre partition comprenne $Z$ » $C^{\prime}$ est très matériel, très terre-à-terre. Et si l'élève ne vous croit pas, vous faites jouer son œuvre et là, il s'aperçoit luimême de ses erreurs; au fond, c'est la meilleure pédagogie.

Ceci dit, mon enseignement je le conçois ainsi : les élèves de composition font tous de l'analyse. Car le meilleur moyen d'apprendre - et on dit que c'est une chose qui ne s'apprend pas -, le meilleur moyen d'apprendre quand même sur les techniques de la composition et sur la composition, c'est de 
passer par l'analyse des œuvres: examiner les problèmes que les compositeurs $d$ 'hier et d'aujourd'hui ont eus et comment ils les ont résolus. Alors, j'analyse des œuvres de toutes les époques; (et ça, i'y tiens beaucoup) depuis le chant grégorien jusqu'à la musique contemporaine en passant par les grands classiques, et également avec quelques incursions dans les musiques extraoccidentales. C'est à travers l'analyse des œuvres que l'esprit des compositeurs s'éveille aux réalités musicales; et en même temps, ça constitue une sorte de terrain, d'humus extrêmement riche comme source d'inspiration pour leurs propres œuvres. II est arrivé une fois, alors que j'analysais un concerto de Mozart, que quelqu'un m'a dit que ça ne l'intéressait pas. Je lui ai répondu qu'il n'était pas obligé de rester. Mais moi, je trouve qu'il est essentiel de connaîre un des plus grands inventeurs de formes de l'histoire; et puis, au point de vue de l'accentuation des phrases, cette musique est d'une richesse singulière; il y a des musiques bien postérieures à Mozart, très près de nous même, qui ne vont pas au quart de la variété rythmique que nous enseignent ses œuvres.

Toutes ces analyses, je ne les fais pas dans une perspective historique, c'est-à-dire que je ne commence pas à l'époque de Mathusalem pour arriver à aujourd'hui; je mélange les époques, les genres, selon les besoins des élèves. J'ai beaucoup analysé, par exemple, Monteverdi. Ça m'a amené à étudier le rapport texte-musique. À partir d'une œuvre de Monteverdi, je peux très bien sauter dans une œuvre vocale de Berg ou de Debussy ou encore dans du Berio où ce sont des phonèmes qui sont utilisés /dans Monteverdi, on trouve d'ailleurs des embryons de choses comme ça).

Je poursuis ainsi avec les élèves une sorte d'aventure: on travaille ensemble. Bien sûr, je suis le professeur; mais pour les élèves, je suis surtout un guide. Quand ils apportent des pièces, je pose des questions par lesquelles j'essaie de les aider à voir plus clair dans ce qu'ils font. Au point de vue style, j'interviens le moins possible, sauf si quelqu'un arrive avec une œuvre qui ne contient que des clichés: ça, honnêtement, je pense que les élèves doivent en prendre conscience.

D'autre part, je serais très malheureux que mes élèves soient influencés par mes œuvres et qu'ils fassent des choses qui ressemblent à ce que je fais. Ça arrive de temps à autre, et ça me met mal à l'aise. Ceci dit, face à la question du style, il faut faire abstraction de ses goûts personnels, dans la mesure du possible : les élèves vont dans une certaine direction, explorent des routes qui, parfois, me sont totalement étrangères. Je les encourage beaucoup dans leur démarche; c'est une question de respect, et c'est dans ce sens-là qu'on ne 
peut pas enseigner la composition. On peut toutefois travailler comme dans un atelier, comme dans ces ateliers du Moyen Âge et de la Renaissance où les apprentis travaillaient avec un maître: chacun cherchait et faisait part de ses découvertes.

\section{Grandeurs et misères de la musique contemporaine}

Ce que je vais dire, je le dis sans geindre, quoique ça puisse ressembler à ça: nous n'existons pratiquement pas. Et ce, bien qu'il y ait à Montréal une Société de musique contemporaine qui existe depuis plus de quinze ans et qui a une activité dont l'intérêt se compare à celui de groupements semblables dans les autres grandes villes nord-américaines et européennes: on y a joué des œuvres du monde entier. Vraiment, la Société de musique contemporaine est un foyer qui, depuis sa fondation, a toujours voulu une ouverture au monde: on y a créé et joué des musiques d'ici en les confrontant avec des musiques américaines, européennes et japonaises. Cette ouverture a été très saine : comme quand on ouvre les fenêtres, ça a fait entrer de l'oxygène. Bon, ça existe, c'est magnifique, c'est une chose qui doit se développer sous diverses formes, mais ça reste quand même, vis-à-vis des media, marginal. C'est Heidegger qui dit: "Où est l'événement important $\zeta$ ». Est-ce que c'est la manchette du journal du jour ou est-ce l'invention du scientifique qui a lieu le même jour et dont personne n'a entendu parler ¿ Ou la naissance d'un poème ? Du point de vue journalistique, du quotidien, c'est la manchette; mais du point de vue historique, ce n'est peut-être pas la manchette qui sera oubliée dans quelques semaines, mais plutôt l'invention, l'œuvre d'art, qui feront leur chemin. Cette situation marginale de la musique contemporaine est un état de fait qu'on est bien obligé de constater, mais non pas d'accepter. Je me souviens lorsque Serge Garant, François Morel et moi-même avons organisé, au Conservatoire, le premier concert de musique contemporaine à Montréal (nous étions jeunes étudiants à ce moment-là): $c^{\prime}$ était le $1^{\text {er }}$ mai 1954; on a joué nos œuvres et des œuvres de Webern, Messiaen, Boulez. Le lendemain, il y a un journal qui, dans sa critique, a parlé de musique communiste. Pour n'être pas trop méchant, attribuons cela à l'effet de pure coïncidence, le concert ayant eu lieu le jour de la Fête des travailleurs. (Notre geste n'avait évidemment rien à voir avec cette manifestation). J'ai choisi cet exemple pour montrer la distance qu'il pouvait y avoir, à ce moment-là, entre ce que nous faisions et la façon dont c'était reçu. II y a eu, bien sûr, un progrès depuis, et je pense qu'il faut mentionner très fort ici le rôle d'un homme comme Garant dans le milieu musical. En tant que compositeur d'abord: c'est 
un compositeur très important qui, à mon avis, n'est pas apprécié à sa juste valeur maintenant, ce qui est peut-être un bon signe sur la valeur de son œuvre. Puis, en tant qu'interprète: là, on a affaire à la générosité même; Garant s'est très souvent effacé pour jouer les autres, pour créer les œuvres des autres compositeurs, de ses collègues et des plus jeunes. Il y en a d'autres qui ont travaillé à ce progrès mais je veux spécialement mentionner le nom de Serge Garant. L'activité de la SMCQ a eu une incidence très importante dans le milieu: il y a eu des ateliers qui se sont formés; il y a eu des groupes comme Les Événements du Neuf qui sont nés après. Ils ne sont pas nés à cause de la SMCQ directement, mais si la SMCQ n'avait pas existé, est-ce que ces groupes auraient pu se développer $\$ Non seulement ça, mais un certain savoir-faire des musiciens de l'Orchestre symphonique de Montréal c'était souvent les mêmes musiciens qui jouaient à la SMCQ et à l'OSM vient du travail qu'ils ont fait en musique contemporaine à la $S M C Q$; ce qui fait que quelqu'un comme Charles Dutoit peut maintenant monter en peu de temps une œuvre de Xenakis. Ça, c'est une chose qui n'est écrite nulle part, qui ne se mesure pas, mais qui est très tangible. 


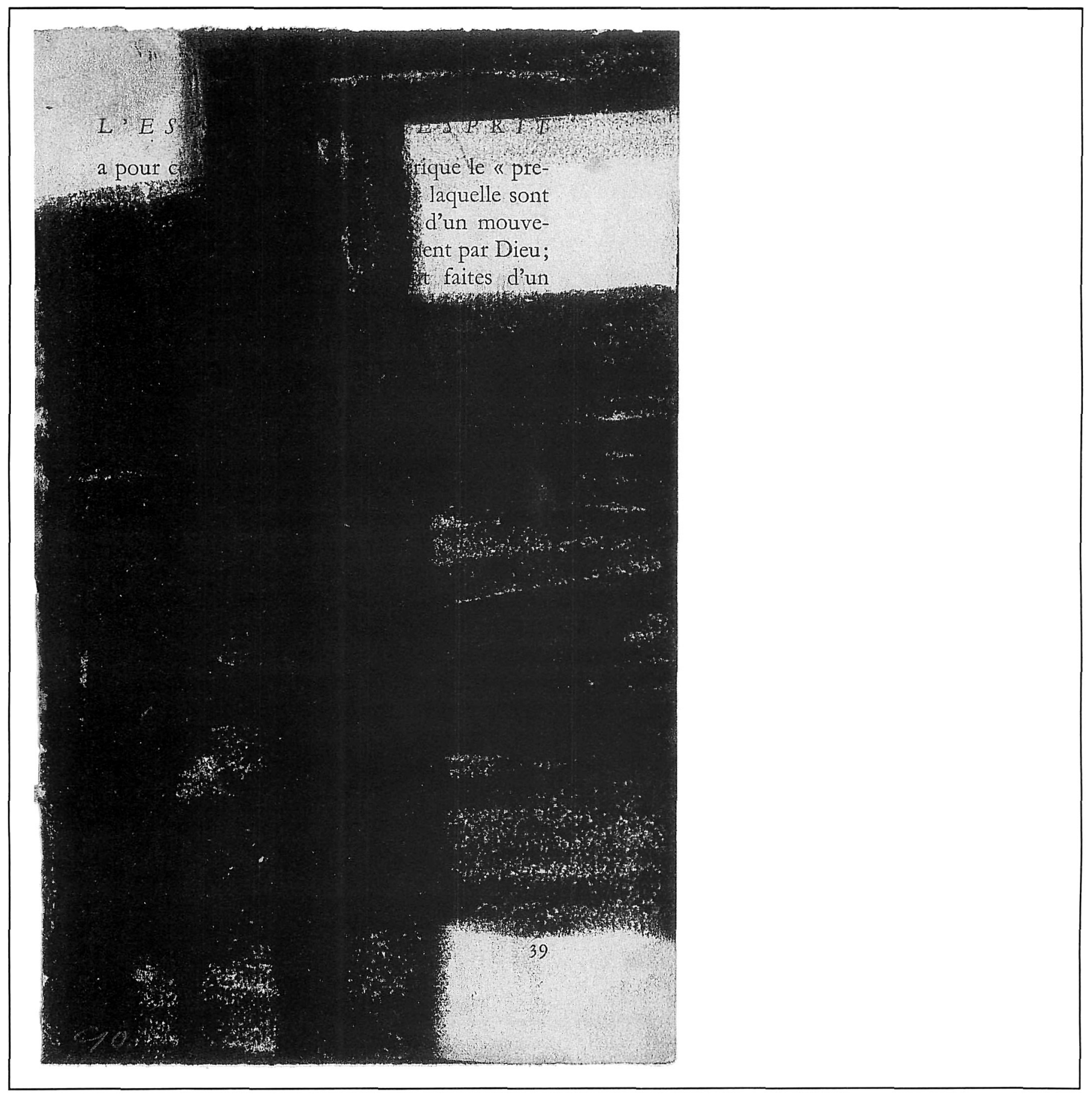

